\title{
The pleural mesothelium in development and disease
}

\section{Hitesh Batra* and Veena B. Antony}

Division of Pulmonary, Allergy and Critical Care Medicine, Department of Medicine, University of Alabama at Birmingham, Birmingham, AL, USA

\section{Edited by:}

Sotirios G. Zarogiannis, University of Thessaly, Greece

\section{Reviewed by:}

Vassilios Liakopoulos, Aristotle University of Thessaloniki, Greece K. Onuma, Juntendo University, Japan

\section{*Correspondence}

Hitesh Batra, Division of Pulmonary, Allergy and Critical Care Medicine, Department of Medicine, University of Alabama at Birmingham, 1900

University B/vd., THT 422,

Birmingham, AL 35294-0006, USA e-mail:hiteshbatra921@gmail.com
The pleural mesothelium, derived from the embryonic mesoderm, is formed by a metabolically active monolayer of cells that blanket the chest wall and lungs on the parietal and visceral surfaces, respectively. The pleura and lungs are formed as a result of an intricate relationship between the mesoderm and the endoderm during development. Mesenchymal signaling pathways such as Wnt/B-catenin, Bmp4, and sonic hedgehog appear to be quintessential for lung development. Pleural Mesothelial Cells (PMCs) are known to express Wilms tumor-1 (Wt1) gene and in lineage labeling studies of the developing embryo, PMCs were found to track into the lung parenchyma and undergo mesothelial-mesenchymal transition (MMT) to form $\alpha$-smooth muscle actin

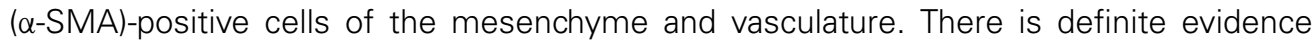
that mesothelial cells can differentiate and this seems to play an important role in pleural and parenchymal pathologies. Mesothelial cells can differentiate into adipocytes, chondrocytes, and osteoblasts; and have been shown to clonally generate fibroblasts and smooth muscle cells in murine models. This supports the possibility that they may also modulate lung injury-repair by re-activation of developmental programs in the adult reflecting an altered recapitulation of development, with implications for regenerative biology of the lung. In a mouse model of lung fibrosis using lineage-tracing studies, PMCs lost their polarity and cell-cell junctional complexes, migrated into lung parenchyma, and underwent phenotypic transition into myofibroblasts in response to the pro-fibrotic mediator, transforming growth factor- $\beta 1$ (TGF- $\beta 1$ ). However, intra-pleural heme-oxygenase-1 ( $\mathrm{HO}-1)$ induction inhibited PMC migration after intra-tracheal fibrogenic injury. Intra-pleural fluorescein isothiocyanate labeled nanoparticles decorated with a surface antibody to mesothelin, a surface marker of mesothelial cells, migrate into the lung parenchyma with PMCs supporting a potential role for pleural based therapies to modulate pleural mesothelial activation and parenchymal disease progression.

\section{Keywords: pleural mesothelium, pleural mesothelial cells, idiopathic pulmonary fibrosis (IPF), Wilms tumor-1} (WT1), epithelial-mesenchymal transition (EMT)

\section{INTRODUCTION}

The lung is a complex, integrated structure of airways, vasculature and interstitium, surrounded by the pleural mesothelium (Jantz and Antony, 2006). The pleural mesothelium, derived from the embryonic mesoderm, is formed by a layer of cells that blanket the chest wall and lungs on the parietal and visceral surfaces, respectively. A complex interplay of signaling pathways such as Wnt/Bcatenin, Bone morphogenetic protein 4 (Bmp4), sonic hedgehog, and Fibroblast growth factor 10 (Fgf10) between the developing endoderm and mesoderm is essential for development.

Wilms tumor-1 (Wt1), a zinc finger transcription factor (Haber et al., 1990), is expressed in certain mesoderm-derived

Abbreviations: PMCs, Pleural Mesothelial Cells (PMCs); Wt1, Wilms tumor-1 (Wt1); MMT, Mesothelial-mesenchymal transition (MMT); $\alpha$-SMA, $\alpha$-smooth muscle actin; Bmp4, Bone morphogenetic protein 4; Shh, Sonic hedgehog; Fgf10, Fibroblast growth factor 10; IPF, Idiopathic Pulmonary Fibrosis; TGF$\beta 1$, Transforming growth factor- $\beta 1$; HO-1, Heme-oxygenase-1 (HO-1); EMT, Epithelial-mesenchymal transition; NOX-4, NADPH oxidase-4; UIP, Usual interstitial pneumonia; RNA, Ribonucleic acid; BAL, Bronchoalveolar lavage; NP, Nanoparticle; FITC, Fluorescein isothiocyanate; PLGA, Poly-lactic-co-glycolic acid. tissues including the pleura (Park et al., 1993) and is known to regulate many functional properties of the developing mesothelium (Ito et al., 2006; Jomgeow et al., 2006). It also regulates pleural mesothelial cell (PMC) plasticity. Wt1 expressing PMCs have been shown to migrate into the lung parenchyma and differentiate into subpopulations of bronchial smooth muscle cells, vascular smooth muscle cells and fibroblasts (Colvin et al., 2001; Dixit et al., 2013).

The presence of Wt1-expressing PMCs on both the pleural surface and in the lung parenchyma of patients with IPF suggests a role for crosstalk between the parenchymal lung injury and activation of the pleural mesothelium in the pathogenesis of IPF. PMCs migrate into the pulmonary parenchyma in IPF and transition into myofibroblasts suggesting the novel hypothesis that IPF is an altered, recapitulation of development, with implications for regenerative biology of the lung.

PMCs could represent a new cellular therapeutic target in a disease where we currently lack treatment modalities. Intra-pleural delivery of compounds is a lung targeted, innovative 
therapeutic modality that can be refined to deliver drugs, minimizing systemic toxicity.

\section{DEVELOPMENT}

The lungs arise from the anterior foregut endoderm via a complex and highly regulated process comprising of the embryonic stage, pseudo-glandular stage, branching morphogenesis, canalicular stage, saccular stage, and the alveolarization stage. During all stages of endodermal development, the lung mesoderm interacts with the lung endoderm to generate the various lineages within the lung (Herriges and Morrisey, 2014) and plays a central role in regulating morphogenesis of the lung, including branching, lung size, and vascular development (Que et al., 2008).

\section{ROLE OF MESODERM DURING DEVELOPMENT}

The lung mesoderm is an important source of paracrine signals such as Fgf10 and Wnt2 (Bellusci et al., 1997; Sekine et al., 1999; Weaver et al., 2000; Goss et al., 2009), which are essential for multiple processes during lung development, including the patterning of early endoderm progenitors, epithelial proliferation, and differentiation. The mature lung contains many mesodermal derivatives, including airway smooth muscle, vascular smooth muscle, endothelial and mesothelial cells, as well as multiple less well-understood cell types, such as pericytes, alveolar fibroblasts, and lipofibroblasts (Herriges and Morrisey, 2014). In lineage labeling studies of the developing embryo, PMCs were found to track into the lung parenchyma and undergo mesothelial-mesenchymal transition (MMT) to form $\alpha$-smooth muscle actin ( $\alpha$-SMA)positive cells of the mesenchyme and vasculature (Que et al., 2008).

\section{SIGNALING PATHWAYS}

During development, Wnt/ $\beta$-catenin signaling is responsible for specifying Nkx2.1+ respiratory endoderm progenitors, which mark the specification of the respiratory system in the anterior foregut endoderm (Herriges and Morrisey, 2014). This, in turn, is dependent upon active Bmp signaling that represses the transcription factor Sox2, thereby allowing for expression of $\mathrm{Nkx2}$.1. Loss of Bmp signaling through inactivation of the Bmp receptors, Bmprla and Bmprlb, leads to tracheal agenesis with retention of the branching region of the lungs (Domyan et al., 2011). Moreover, signaling between the developing endoderm and mesoderm appears to be essential for branching morphogenesis and the loss of Fgf10 signaling to Fgfr2 in the developing endoderm leads to disruption of branching (Sekine et al., 1999; Ohuchi et al., 2000). Fgf10 expression is regulated by several signaling pathways such as Bmp4 and sonic hedgehog (Shh), suggesting a complex interplay of signaling molecules during development (Bellusci et al., 1997; Pepicelli et al., 1998; Weaver et al., 2000).

\section{Wt1}

The Wt1 gene encodes a $49-52 \mathrm{kDa}$ protein with an $\mathrm{N}$-terminal domain that is involved in protein-RNA interactions critical for its transcriptional regulatory function (Call et al., 1990).

\section{Wt1 IN PRENATAL DEVELOPMENT}

PMCs are known to express Wt1 gene and migrate into the lung parenchyma to form smooth muscle cells of the vascular wall, as well as other cells of the lung mesenchyme during development (Que et al., 2008; Zhou et al., 2010, 2011).

Recent cell lineage labeling studies in the developing heart provide evidence that the surface epicardial mesothelium undergoes epithelial-mesenchymal transition (EMT) and migrates into the myocardium where it differentiates into various cell types, including endothelium, smooth muscle cells, and cardiomyocytes (Mikawa and Gourdie, 1996; Dettman et al., 1998; Cai et al., 2008; Zhou et al., 2010). In addition, lineage tracing and other studies show that the serosal mesothelium of the gut also contributes the majority of vascular smooth muscle cells (Wilm et al., 2005; Kawaguchi et al., 2007). A major role of Wt1 and PMCs in mesenchymal differentiation and development was demonstrated by lineage tracing studies in the embryonic mouse (Colvin et al., 2001). PMCs were shown to readily migrate into the lung parenchyma and express $\alpha$-SMA. Another recent study employing $\mathrm{Wt} 1^{\mathrm{CreERT} 2 /+}$ mice visualized $\mathrm{Wt} 1+$ mesothelial cell entry into the lung by live imaging, identified their progenies in subpopulations of bronchial smooth muscle cells, vascular smooth muscle cells, and desmin + fibroblasts by lineage tagging; and demonstrated that mesothelial cell movement into the lung requires the direct action of sonic hedgehog (shh) signaling (Dixit et al., 2013). Together, these studies demonstrate that the mesothelium lining of serosal surfaces of multiple organ systems plays a critical role during development and organogenesis, supporting the possibility that they may also modulate lung injury-repair by re-activation of developmental programs in the adult.

\section{Wt1 IN POSTNATAL DISEASE}

Wt1 can induce a morphological transition from an epithelial phenotype to a mesenchymal phenotype (Burwell et al., 2007). It is a potent transcription factor that can function as a tumor suppressor (Zhang et al., 2003), or as an oncogene depending on the cell type (Loeb et al., 2001; Oji et al., 2002; Ueda et al., 2003). It was in fact initially discovered as a tumor suppressor gene in Wilms tumor of the kidney (Haber et al., 1990). Wt1 demonstrates tissue specific responses and is recognized to regulate TGF- $\beta 1$ in the kidney. In other cell types such as hematological cells it is reported to confer oncogenic properties (Licciulli and Kissil, 2010). Wt1 is expressed in normal pleural mesothelium, and is over-expressed in malignant mesothelioma (Amin et al., 1995). However, there is limited information of the role of Wt1 in non-malignant lung diseases.

\section{Wt1 IN IDIOPATHIC PULMONARY FIBROSIS (IPF)}

Several studies support a role for Wt1 in mesothelial-tomesenchymal transition (MMT) (Burwell et al., 2007; Bax et al., 2011a,b). Hecker et al. have evaluated the responses of PMCs to TGF- $\beta 1$, their expression of MMT markers such as e-cadherin, $\alpha$-SMA, vimentin; their contractile ability using a collagen gel contraction assay (Hecker et al., 2009); and also biological functional assays such as migration and proliferation (Hecker et al., 2009). Studies indicate a role for TGF- $\beta 1$ in mediating MMT (Nasreen et al., 2009). For example, treatment of mouse 
PMCs with TGF- $\beta 1$ increased expression of the myofibroblast markers $\alpha$-SMA and NADPH oxidase-4 (NOX-4) (Zolak et al., 2013). Wt1-expressing PMCs are present both on the pleural surface and in the lung parenchyma of patients with IPF suggesting their role in the pathogenesis of IPF. However, the precise relationship between $\mathrm{Wt} 1$ and TGF- $\beta 1$ remains unclear. Also, the role of $\mathrm{Wt} 1$ in regulating PMC plasticity needs to be analyzed.

\section{PLURIPOTENCY OF MESOTHELIAL CELLS}

Although mesenchymal in origin, mesothelial cells exhibit characteristics such as a polygonal cell shape, expression of surface microvilli, epithelial cytokeratins, and tight junctions, which are typical of epithelial cells (Mutsaers, 2002). During development, endothelium and vascular smooth muscle cells of the vascular system, heart, liver and gut are derived from the differentiation of mesothelium via a process of epithelial-to-mesenchymal transition (EMT) (Munoz-Chapuli et al., 1999; Perez-Pomares et al., 2002, 2004). Increasing evidence suggests existence of a population of progenitor-like mesothelial cells with the capacity to differentiate into cells of different phenotypes (Herrick and Mutsaers, 2004). By targeting mesothelin and using a genetic lineage tracing approach, Rinkevich et al. demonstrated that embryonic and adult mesothelium represents a common lineage to trunk fibroblasts, smooth muscle cells, and vasculature (Rinkevich et al., 2012).

Recently, primary rat and human mesothelial cells, when maintained in osteogenic or adipogenic media, were shown to differentiate into osteoblast- and adipocyte-like cells via epithelial-to-mesenchymal transition as suggested by the changes in mRNA expression of these cells. This supports mesothelial cell differentiation as the potential source of different tissue types in malignant mesothelioma and other serosal pathologies (Lansley et al., 2011). The term mesodermoma was introduced by Donna and Betta to define neoplasms arising from undifferentiated and multipotential mesoderm (Donna and Betta, 1981).

Mesothelial cells appear to retain the ability to produce mesenchyme, including smooth muscle cells, in response to transforming growth factor- $\beta_{1}$ (TGF- $\left.\beta_{1}\right)$ and platelet derived growth factor (Wada et al., 2003; Kawaguchi et al., 2007). Moreover, mesothelial cells have been shown to adopt a myofibroblast phenotype in vitro, in response to TGF- $\beta_{1}$ (Yang et al., 2003). Transfection of the peritoneum and pleura of rats with an adenovirus expressing TGF- $\beta_{1}$ induced mesothelial cells to undergo EMT with subsequent fibrotic changes (Margetts et al., 2005; Decologne et al., 2007).

The significance of EMT in malignant pleural mesothelioma (MPM) was demonstrated by Schramm et al. in their study of a retrospective cohort of 352 patients. Immunohistochemistry of a tissue microarray showed that the activation of periostintriggered EMT is associated with the sarcomatoid histotype of malignant mesothelioma and has an impact on shorter survival of patients (Schramm et al., 2010). Periostin secretion by MPM cells has in turn been shown to be upregulated by CD26 (Komiya et al., 2014), the expression of which is increased in various cancers (Havre et al., 2008).

\section{IDIOPATHIC PULMONARY FIBROSIS (IPF) SUB-PLEURAL DISTRIBUTION}

IPF is a progressive lung disease process that appears to begin in the sub-pleural regions, and then extend centrally. This subpleural and basilar distribution, which is the defining feature of idiopathic pulmonary fibrosis (IPF) has not been adequately explained (King et al., 2011). However, this pattern of distribution suggests pleural involvement in the disease process.

\section{MYOFIBROBLASTS AND FIBROBLASTIC FOCI}

The presence of myofibroblasts in fibrobastic foci in lungs of patients with IPF is well-established. The fibroblast and myofibroblast foci secrete excessive amounts of extracellular matrix, mainly collagens, resulting in scarring and destruction of the lung architecture. The profusion of fibroblastic foci is predictive of survival in IPF patients (King et al., 2001a,b). A recent study has shown that the hallmark lesion in usual interstitial pneumonia (UIP), fibroblastic foci, on three-dimensional histo-pathological reconstruction are part of a complex and highly interconnected reticulum of fibrous tissue that extends from the pleura into the lung parenchyma (Cool et al., 2006). Neither the origin of these myofibroblasts nor the molecular mechanisms involved in the formation of fibroblastic foci have been well-defined (Phan, 2008).

While a number of cellular source(s) and progenitors of tissue myofibroblasts have been proposed, none of these hypotheses provide a indisputable explanation for the histo-pathological pattern of usual interstitial pneumonia (UIP) and its peripheral localization (Raghu et al., 2011). However, evidence suggests that abnormal recapitulation of developmental pathways and epigenetic changes may play a role in the pathogenesis of IPF (King et al., 2011). IPF is a devastating disease with an inexorable course and its pathogenesis remains unclear. There are no therapies that directly impact the disease progression or mortality, and we urgently need innovative new ideas that will impact therapeutics in this disease (King et al., 2011; Raghu et al., 2011). PMCs serve as myofibroblast progenitors in animal models of fibrosis, and therapeutic targeting of these cells may be effective as an anti-fibrotic approach (Que et al., 2008).

\section{PLEURAL MESOTHELIAL CELLS IN IPF}

PMCs are metabolically active cells (Antony, 2003; Mohammed et al., 2007), responsive to their microenvironment (Antony, 2003), and are recognized to demonstrate plasticity of their phenotype (Nasreen et al., 2009). The presence of calretinin and mesothelin (markers of mesothelial cells) (Mubarak et al., 2012) and Wt-1 expressing PMCs (Zolak et al., 2013) was recently demonstrated in parenchymal cells of explanted lung tissues from 16 patients with IPF supporting a role for PMC differentiation and their trafficking into the lung as contributors to the myofibroblast population in lung fibrosis. It is not known if the number or profusion of PMCs may be predictive of the severity and/or progression of IPF.

The Ashcroft score is a score for histo-pathological grading of pulmonary fibrosis (Hagiwara et al., 2000; Matsuoka et al., 2002; Simler et al., 2002; Murakami et al., 2006). There exists a correlation between the number of calretinin-positive cells and the 
degree of fibrotic change in the parenchyma, as measured by the Ashcroft score. Whether the number of calretinin-positive cells was measured as a raw number, or as percentage of the total nucleated cells seen in a photomicrograph, the correlation with the degree of fibrosis was highly significant (Mubarak et al., 2012). These studies suggest that PMCs may represent a novel cellular biomarker of disease activity, and more importantly, play a role in the tissue remodeling responses seen in patients with IPF.

Recent studies demonstrate that PMCs cells lose their polarity and cell-cell junctional complexes, migrate into lung parenchyma, and undergo phenotypic transition into myofibroblasts in response to the pro-fibrotic mediator, TGF- $\beta 1$ (Nasreen et al., 2009; Mubarak et al., 2012). The transition of PMC to myofibroblasts is dependent on smad-2 signaling and knockdown of smad-2 gene by silencing small interfering RNA significantly suppresses the transition of PMCs to myofibroblasts and inhibits PMC haptotaxis (Nasreen et al., 2009). We have demonstrated in a murine model, that TGF- $\beta 1$ induces PMC trafficking into the lung and differentiation into myofibroblasts. Moreover, carbon monoxide or the induction of heme oxygenase-1 (HO-1) inhibits the expression of myofibroblast markers, contractility, and haptotaxis in PMCs treated with TGF- $\beta 1$. These findings support a potential role for pleural-based therapies to modulate pleural mesothelial activation and parenchymal fibrosis progression (Zolak et al., 2013).

\section{PLEURAL MESOTHELIAL CELLS AS A POTENTIAL THERAPEUTIC TARGET}

PMCs migrate into the pulmonary parenchyma in IPF and transition into myofibroblasts. This invokes a novel, alternative hypothesis for the origin and source of the myofibroblasts in the lungs of patients with IPF, and provides a rational explanation for the spatio-temporal distribution of fibrosis in IPF. PMCs could represent a new cellular therapeutic target in a disease where we currently lack treatment options. Intra-pleural delivery of compounds is a lung targeted, innovative therapeutic modality that can be refined to deliver drugs, minimizing systemic toxicity.

Direct delivery of the small molecule inhibitors to the pleura could potentially have several advantages: (Jantz and Antony, 2006) It can potentially provide a direct, high concentration of the compound to target the pro-fibrogenic activities of PMCs; (Haber et al., 1990) Intra-pleural delivery might delay systemic absorption, protecting against systemic toxicity while increasing efficacy; (Park et al., 1993) Intra-pleural delivery may result in higher, sustained levels in the Bronchoalveolar lavage (BAL) when compared with serum levels (Mubarak et al., 2012); and (Jomgeow et al., 2006) innovative, less invasive methods of accessing the pleural space such as tunneled pleural catheters could make intra-pleural delivery a viable option in patients.

There are several methods that can be utilized for sitedirected delivery of therapeutic agents. These include liposomal drug delivery, nanoparticle (NP) delivery of proteins, and gene therapy (Watanabe et al., 2010). Several of these methods have been utilized to target the pleura (Perez-Soler et al., 1997; Liu et al., 2006; Watanabe et al., 2010). We have demonstrated that PMCs migrate into the parenchyma (Zolak et al., 2013). Biodegradable fluorescein isothiocyanate (FITC) labeled
PLGA (poly-lactic-co-glycolic acid) nanoparticles (which can carry therapeutic compounds conjugated to PLGA) decorated with antibody targeted to mesothelin (a PMC marker) localize to the pleural surface in control mice, but diffuse into the lung parenchyma in mice given intra-tracheal bleomycin (Figure 1). It appears that intra-pleural delivery of molecules to the lung is feasible, albeit, with the recognition that refinement of techniques for minimal lung injury will be required.

\section{CONCLUSION}

The pleural mesothelium is a derivative of the embryonic mesoderm. Several complex pathways of interplay between the mesoderm and endoderm lead to the development of the lungs and pleura. Lineage labeling and other studies have shown the ability of the PMCs to undergo MMT to form other mesenchymal structures and have therefore established their pluripotency during development. Moreover, recent studies have shown that the plasticity of mesothelial cells is retained postnatally and gives them the ability to migrate and differentiate into other cell types. This supports the possibility that PMCs may modulate lung injury-repair by reactivation of developmental programs in the adult, reflecting an altered recapitulation of development with implications for regenerative biology of the lung. We have shown that PMCs are present in the lung parenchyma in lungs with IPF. Also, PMC migration into lung parenchyma and phenotypic transition into myofibroblasts can be induced by the pro-fibrotic mediator, TGF- $\beta 1$ and is inhibited by intra-pleural heme-oxygenase-1 (HO-1). The possible role of PMCs in disease pathogenesis and/or progression of IPF makes them an attractive cellular target for potential therapeutic interventions in a disease where we currently lack treatment modalities. Intra-pleural delivery of compounds is a lung targeted, innovative therapeutic modality that can be refined to deliver drugs, thereby minimizing systemic toxicity. Intra-pleural fluorescein isothiocyanate labeled nanoparticles decorated with a surface antibody to mesothelin, a surface marker of mesothelial cells, migrate into the lung parenchyma with PMCs supporting a potential role for pleural based therapies to modulate pleural mesothelial activation and parenchymal disease progression. However, further studies are

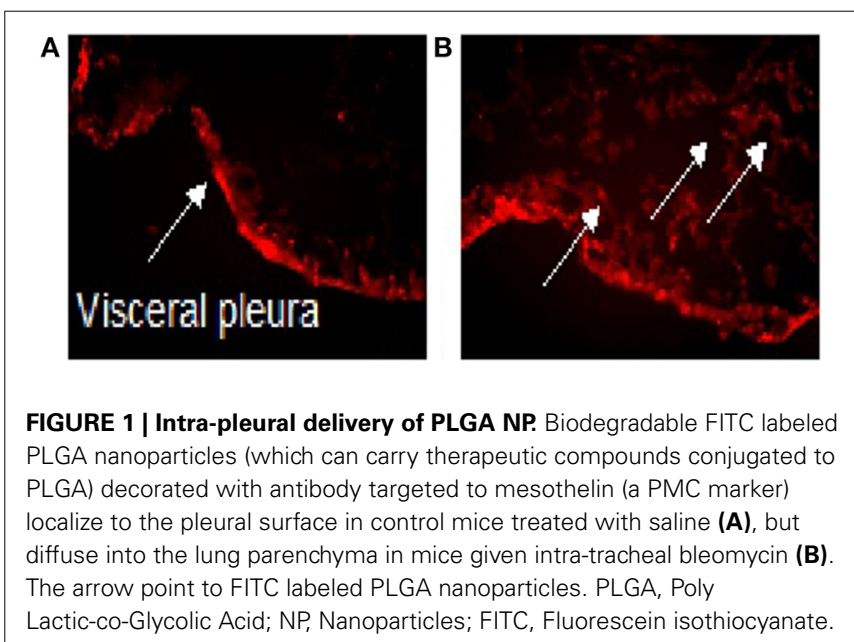


needed to develop such therapies and effective pleural-based drug delivery systems.

\section{REFERENCES}

Amin, K. M., Litzky, L. A., Smythe, W. R., Mooney, A. M., Morris, J. M., Mews, D. J., et al. (1995). Wilms' tumor 1 susceptibility (WT1) gene products are selectively expressed in malignant mesothelioma. Am. J. Pathol. 146, 344-356.

Antony, V. B. (2003). Immunological mechanisms in pleural disease. Eur Respir. J. 21, 539-544. doi: 10.1183/09031936.03.00403902

Bax, N. A., Pijnappels, D. A., van Oorschot, A. A., Winter, E. M., de Vries, A. A., van Tuyn, J., et al. (2011a). Epithelial-to-mesenchymal transformation alters electrical conductivity of human epicardial cells. J. Cell. Mol. Med. 15, 2675-2683. doi: $10.1111 /$ j.1582-4934.2011.01266.x

Bax, N. A., van Oorschot, A. A., Maas, S., Braun, J., van Tuyn, J., de Vries, A. A., et al. (2011b). In vitro epithelial-to-mesenchymal transformation in human adult epicardial cells is regulated by TGFbeta-signaling and WT1. Basic Res. Cardiol. 106, 829-847. doi: 10.1007/s00395-0110181-0

Bellusci, S., Furuta, Y., Rush, M. G., Henderson, R., Winnier, G., and Hogan, B. L. (1997). Involvement of Sonic hedgehog (Shh) in mouse embryonic lung growth and morphogenesis. Development 124, 53-63.

Burwell, E. A., McCarty, G. P., Simpson, L. A., Thompson, K. A., and Loeb, D. M. (2007). Isoforms of Wilms' tumor suppressor gene (WT1) have distinct effects on mammary epithelial cells. Oncogene 26, 3423-3430. doi: 10.1038/sj.onc. 1210127

Cai, C. L., Martin, J. C., Sun, Y., Cui, L., Wang, L., Ouyang, K., et al. (2008). A myocardial lineage derives from Tbx18 epicardial cells. Nature 454, 104-108. doi: 10.1038 /nature 06969

Call, K. M., Glaser, T., Ito, C. Y., Buckler, A. J., Pelletier, J., Haber, D. A., et al. (1990). Isolation and characterization of a zinc finger polypeptide gene at the human chromosome 11 Wilms' tumor locus. Cell 60, 509-520. doi: 10.1016/00928674(90)90601-A

Colvin, J. S., White, A. C., Pratt, S. J., and Ornitz, D. M. (2001). Lung hypoplasia and neonatal death in Fgf9-null mice identify this gene as an essential regulator of lung mesenchyme. Development 128, 2095-2106.

Cool, C. D., Groshong, S. D., Rai, P. R., Henson, P. M., Stewart, J. S., and Brown, K. K. (2006). Fibroblast foci are not discrete sites of lung injury or repair: the fibroblast reticulum. Am. J. Respir. Crit. Care Med. 174, 654-658. doi: 10.1164/rccm.200602-205OC

Decologne, N., Kolb, M., Margetts, P. J., Menetrier, F., Artur, Y., Garrido, C., et al. (2007). TGF-betal induces progressive pleural scarring and subpleural fibrosis. J. Immunol. 179, 6043-6051. doi: 10.4049/jimmunol.179.9.6043

Dettman, R. W., Denetclaw, W. Jr., Ordahl, C. P., and Bristow, J. (1998). Common epicardial origin of coronary vascular smooth muscle, perivascular fibroblasts, and intermyocardial fibroblasts in the avian heart. Dev. Biol. 193, 169-181. doi: 10.1006/dbio.1997.8801

Dixit, R., Ai, X., and Fine, A. (2013). Derivation of lung mesenchymal lineages from the fetal mesothelium requires hedgehog signaling for mesothelial cell entry. Development 140, 4398-4406. doi: 10.1242/dev.098079

Domyan, E. T., Ferretti, E., Throckmorton, K., Mishina, Y., Nicolis, S. K., and Sun, X. (2011). Signaling through BMP receptors promotes respiratory identity in the foregut via repression of Sox2. Development 138, 971-981. doi: 10.1242/dev.053694

Donna, A., and Betta, P. G. (1981). Mesodermomas: a new embryological approach to primary tumours of coelomic surfaces. Histopathology. 5, 31-44. doi: 10.1111/j.1365-2559.1981.tb01764.x

Goss, A. M., Tian, Y., Tsukiyama, T., Cohen, E. D., Zhou, D., Lu, M. M., et al. (2009). Wnt $2 / 2 \mathrm{~b}$ and beta-catenin signaling are necessary and sufficient to specify lung progenitors in the foregut. Dev. Cell 17, 290-298. doi: 10.1016/j.devcel.2009.06.005

Haber, D. A., Buckler, A. J., Glaser, T., Call, K. M., Pelletier, J., Sohn, R. L., et al. (1990). An internal deletion within an 11 p13 zinc finger gene contributes to the development of Wilms' tumor. Cell 61, 1257-1269. doi: 10.1016/00928674(90)90690-G

Hagiwara, S. I., Ishii, Y., and Kitamura, S. (2000). Aerosolized administration of $\mathrm{N}$-acetylcysteine attenuates lung fibrosis induced by bleomycin in mice. Am. J. Respir. Crit. Care Med. 162, 225-231. doi: 10.1164/ajrccm.162.1.99 03129
Havre, P. A., Abe, M., Urasaki, Y., Ohnuma, K., Morimoto, C., and Dang, N. H. (2008). The role of CD26/dipeptidyl peptidase IV in cancer. Front. Biosci. 13, 1634-1645. doi: 10.2741/2787

Hecker, L., Vittal, R., Jones, T., Jagirdar, R., Luckhardt, T. R., Horowitz, J. C., et al. (2009). NADPH oxidase-4 mediates myofibroblast activation and fibrogenic responses to lung injury. Nat. Med. 15, 1077-1081. doi: 10.1038/nm.2005

Herrick, S. E., and Mutsaers, S. E. (2004). Mesothelial progenitor cells and their potential in tissue engineering. Int. J. Biochem. Cell Biol. 36, 621-642. doi: 10.1016/j.biocel.2003.11.002

Herriges, M., and Morrisey, E. E. (2014). Lung development: orchestrating the generation and regeneration of a complex organ. Development 141, 502-513. doi: $10.1242 /$ dev.098186

Ito, K., Oji, Y., Tatsumi, N., Shimizu, S., Kanai, Y., Nakazawa, T., et al. (2006). Antiapoptotic function of 17AA(+)WT1 (Wilms' tumor gene) isoforms on the intrinsic apoptosis pathway. Oncogene 25, 4217-4229. doi: 10.1038/sj.onc. 1209455

Jantz, M. A., and Antony, V. B. (2006). Pleural fibrosis. Clin. Chest Med. 27, 181-191. doi: 10.1016/j.ccm.2005.12.003

Jomgeow, T., Oji, Y., Tsuji, N., Ikeda, Y., Ito, K., Tsuda, A., et al. (2006). Wilms' tumor gene WT1 17AA(-)/KTS(-) isoform induces morphological changes and promotes cell migration and invasion in vitro. Cancer Sci. 97, 259-270. doi: 10.1111/j.1349-7006.2006.00169.x

Kawaguchi, M., Bader, D. M., and Wilm, B. (2007). Serosal mesothelium retains vasculogenic potential. Dev. Dyn. 236, 2973-2979. doi: 10.1002/dvdy.21334

King, T. E. Jr., Pardo, A., and Selman, M. (2011). Idiopathic pulmonary fibrosis. Lancet 378, 1949-1961. doi: 10.1016/S0140-6736(11)60052-4

King, T. E. Jr., Schwarz, M. I., Brown, K., Tooze, J. A., Colby, T. V., Waldron, J. A. Jr., et al. (2001a). Idiopathic pulmonary fibrosis: relationship between histopathologic features and mortality. Am. J. Respir. Crit. Care Med. 164, 1025-1032. doi: 10.1164/ajrccm.164.6.2001056

King, T. E. Jr., Tooze, J. A., Schwarz, M. I., Brown, K. R., and Cherniack, R. M. (2001b). Predicting survival in idiopathic pulmonary fibrosis: scoring system and survival model. Am. J. Respir. Crit. Care Med. 164, 1171-1181. doi: 10.1164/ajrccm.164.7.2003140

Komiya, E., Ohnuma, K., Yamazaki, H., Hatano, R., Iwata, S., Okamoto, T., et al. (2014). CD26-mediated regulation of periostin expression contributes to migration and invasion of malignant pleural mesothelioma cells. Biochem. Biophys. Res. Commun. 447, 609-615. doi: 10.1016/j.bbrc.2014.04.037

Lansley, S. M., Searles, R. G., Hoi, A., Thomas, C., Moneta, H., Herrick, S. E., et al. (2011). Mesothelial cell differentiation into osteoblast- and adipocyte-like cells. J. Cell. Mol. Med. 15, 2095-2105. doi: 10.1111/j.1582-4934.2010.01212.x

Licciulli, S., and Kissil, J. L. (2010). WT1: a weak spot in KRAS-induced transformation. J. Clin. Invest. 120, 3804-3807. doi: 10.1172/JCI44901

Liu, J., Wong, H. L., Moselhy, J., Bowen, B., Wu, X. Y., and Johnston, M. R. (2006). Targeting colloidal particulates to thoracic lymph nodes. Lung Cancer 51, 377-386. doi: 10.1016/j.lungcan.2005.11.006

Loeb, D. M., Evron, E., Patel, C. B., Sharma, P. M., Niranjan, B., Buluwela, L., et al. (2001). Wilms' tumor suppressor gene (WT1) is expressed in primary breast tumors despite tumor-specific promoter methylation. Cancer Res. 61, 921-925.

Margetts, P. J., Bonniaud, P., Liu, L., Hoff, C. M., Holmes, C. J., West-Mays, J. A., et al. (2005). Transient overexpression of TGF- $\{$ beta $\} 1$ induces epithelial mesenchymal transition in the rodent peritoneum. J. Am. Soc. Nephrol. 16, 425-436. doi: 10.1681/ASN.2004060436

Matsuoka, H., Arai, T., Mori, M., Goya, S., Kida, H., Morishita, H., et al. (2002). A p38 MAPK inhibitor, FR-167653, ameliorates murine bleomycin-induced pulmonary fibrosis. Am. J. Physiol. Lung Cell. Mol. Physiol. 283, L103-L112.

Mikawa, T., and Gourdie, R. G. (1996). Pericardial mesoderm generates a population of coronary smooth muscle cells migrating into the heart along with ingrowth of the epicardial organ. Dev. Biol. 174, 221-232. doi: 10.1006/dbio. 1996.0068

Mohammed, K. A., Nasreen, N., and Antony, V. B. (2007). Bacterial induction of early response genes and activation of proapoptotic factors in pleural mesothelial cells. Lung 185, 355-365. doi: 10.1007/s00408-007-9046-6

Mubarak, K. K., Montes-Worboys, A., Regev, D., Nasreen, N., Mohammed, K. A., Faruqi, I., et al. (2012). Parenchymal trafficking of pleural mesothelial cells in idiopathic pulmonary fibrosis. Eur. Respir. J. 39, 133-140. doi: 10.1183/09031936.00141010

Munoz-Chapuli, R., Perez-Pomares, J. M., Macias, D., Garcia-Garrido, L., Carmona, R., and Gonzalez, M. (1999). Differentiation of hemangioblasts 
from embryonic mesothelial cells? A model on the origin of the vertebrate cardiovascular system. Differentiation 64, 133-141. doi: 10.1007/s0025800 50268

Murakami, S., Nagaya, N., Itoh, T., Kataoka, M., Iwase, T., Horio, T., et al. (2006). Prostacyclin agonist with thromboxane synthase inhibitory activity (ONO1301) attenuates bleomycin-induced pulmonary fibrosis in mice. Am. J. Physiol. Lung Cell. Mol. Physiol. 290, L59-L65. doi: 10.1152/ajplung.00042.2005

Mutsaers, S. E. (2002). Mesothelial cells: their structure, function and role in serosal repair. Respirology 7, 171-191. doi: 10.1046/j.1440-1843.2002.00404.x

Nasreen, N., Mohammed, K. A., Mubarak, K. K., Baz, M. A., Akindipe, O. A., Fernandez-Bussy, S., et al. (2009). Pleural mesothelial cell transformation into myofibroblasts and haptotactic migration in response to TGFbetal in vitro. Am. J. Physiol. Lung Cell. Mol. Physiol. 297, L115-L124. doi: 10.1152/ajplung.90587.2008

Ohuchi, H., Hori, Y., Yamasaki, M., Harada, H., Sekine, K., Kato, S., et al. (2000). FGF10 acts as a major ligand for FGF receptor 2 IIIb in mouse multi-organ development. Biochem. Biophys. Res. Commun. 277, 643-649. doi: 10.1006/bbrc. 2000.3721

Oji, Y., Miyoshi, S., Maeda, H., Hayashi, S., Tamaki, H., Nakatsuka, S., et al. (2002). Overexpression of the Wilms' tumor gene WT1 in de novo lung cancers. Int. J. Cancer 100, 297-303. doi: 10.1002/ijc. 10476

Park, S., Schalling, M., Bernard, A., Maheswaran, S., Shipley, G. C., Roberts, D., et al. (1993). The Wilms tumour gene WT1 is expressed in murine mesodermderived tissues and mutated in a human mesothelioma. Nat. Genet. 4, 415-420. doi: 10.1038/ng0893-415

Pepicelli, C. V., Lewis, P. M., and McMahon, A. P. (1998). Sonic hedgehog regulates branching morphogenesis in the mammalian lung. Curr. biol. 8, 1083-1086. doi: 10.1016/S0960-9822(98)70446-4

Perez-Pomares, J. M., Carmona, R., Gonzalez-Iriarte, M., Atencia, G., Wessels, A., and Munoz-Chapuli, R. (2002). Origin of coronary endothelial cells from epicardial mesothelium in avian embryos. Int. J. Dev. Biol. 46, 1005-1013.

Perez-Pomares, J. M., Carmona, R., Gonzalez-Iriarte, M., Macias, D., Guadix, J. A., and Munoz-Chapuli, R. (2004). Contribution of mesothelium-derived cells to liver sinusoids in avian embryos. Dev. Dyn 229, 465-474. doi: 10.1002/dvdy. 10455

Perez-Soler, R., Shin, D. M., Siddik, Z. H., Murphy, W. K., Huber, M., Lee, S. J., et al. (1997). Phase I clinical and pharmacological study of liposome-entrapped NDDP administered intrapleurally in patients with malignant pleural effusions. Clin. Cancer Res. 3, 373-379.

Phan, S. H. (2008). Biology of fibroblasts and myofibroblasts. Proc. Am. Thorac. Soc. 5, 334-337. doi: 10.1513/pats.200708-146DR

Que, J., Wilm, B., Hasegawa, H., Wang, F., Bader, D., and Hogan, B. L. (2008). Mesothelium contributes to vascular smooth muscle and mesenchyme during lung development. Proc. Natl. Acad. Sci. U.S.A. 105, 16626-16630. doi: 10.1073/pnas.0808649105

Raghu, G., Collard, H. R., Egan, J. J., Martinez, F. J., Behr, J., Brown, K. K., et al. (2011). An official ATS/ERS/JRS/ALAT statement: idiopathic pulmonary fibrosis: evidence-based guidelines for diagnosis and management. Am. J. Respir. Crit. Care Med. 183, 788-824. doi: 10.1164/rccm.2009-040GL

Rinkevich, Y., Mori, T., Sahoo, D., Xu, P. X., Bermingham, J. R. Jr., and Weissman, I. L. (2012). Identification and prospective isolation of a mesothelial precursor lineage giving rise to smooth muscle cells and fibroblasts for mammalian internal organs, and their vasculature. Nat. Cell Biol. 14, 1251-1260. doi: $10.1038 /$ ncb2610

Schramm, A., Opitz, I., Thies, S., Seifert, B., Moch, H., Weder, W., et al. (2010). Prognostic significance of epithelial-mesenchymal transition in malignant pleural mesothelioma. Eur. J. Cardiothorac. Surg. 37, 566-572. doi: 10.1016/j.ejcts.2009.08.027

Sekine, K., Ohuchi, H., Fujiwara, M., Yamasaki, M., Yoshizawa, T., Sato, T., et al. (1999). Fgf10 is essential for limb and lung formation. Nat. Genet. 21, 138-141. doi: $10.1038 / 5096$

Simler, N. R., Howell, D. C., Marshall, R. P., Goldsack, N. R., Hasleton, P. S., Laurent, G. J., et al. (2002). The rapamycin analogue SDZ RAD attenuates bleomycin-induced pulmonary fibrosis in rats. Eur Respir. J. 19, 1124-1127. doi: $10.1183 / 09031936.02 .00281602$

Ueda, T., Oji, Y., Naka, N., Nakano, Y., Takahashi, E., Koga, S., et al. (2003) Overexpression of the Wilms' tumor gene WT1 in human bone and soft-tissue sarcomas. Cancer Sci. 94, 271-276. doi: 10.1111/j.1349-7006.2003.tb01432.x

Wada, A. M., Smith, T. K., Osler, M. E., Reese, D. E., and Bader, D. M. (2003). Epicardial/Mesothelial cell line retains vasculogenic potential of embryonic epicardium. Circ. Res. 92, 525-531. doi: 10.1161/01.RES.0000060484.11032.0B

Watanabe, M., Boyer, J. L., and Crystal, R. G. (2010). AAVrh.10-mediated genetic delivery of bevacizumab to the pleura to provide local anti-VEGF to suppress growth of metastatic lung tumors. Gene Ther. 17, 1042-1051. doi: 10.1038/gt.2010.87

Weaver, M., Dunn, N. R., and Hogan, B. L. (2000). Bmp4 and Fgf10 play opposing roles during lung bud morphogenesis. Development 127, 2695-2704.

Wilm, B., Ipenberg, A., Hastie, N. D., Burch, J. B., and Bader, D. M. (2005). The serosal mesothelium is a major source of smooth muscle cells of the gut vasculature. Development 132, 5317-5328. doi: 10.1242/dev.02141

Yang, A. H., Chen, J. Y., and Lin, J. K. (2003). Myofibroblastic conversion of mesothelial cells. Kidney Int. 63, 1530-1539. doi: 10.1046/j.15231755.2003.00861.x

Zhang, T. F., Yu, S. Q., Guan, L. S., and Wang, Z. Y. (2003). Inhibition of breast cancer cell growth by the Wilms' tumor suppressor WT1 is associated with a destabilization of beta-catenin. Anticancer Res. 23, 3575-3584.

Zhou, B., Honor, L. B., He, H., Ma, Q., Oh, J. H., Butterfield, C., et al. (2011). Adult mouse epicardium modulates myocardial injury by secreting paracrine factors. J. Clin. Invest. 121, 1894-1904. doi: 10.1172/JCI45529

Zhou, B., von Gise, A., Ma, Q., Hu, Y. W., and Pu, W. T. (2010). Genetic fate mapping demonstrates contribution of epicardium-derived cells to the annulus fibrosis of the mammalian heart. Dev. Biol. 338, 251-261. doi: 10.1016/j.ydbio.2009.12.007

Zolak, J. S., Jagirdar, R., Surolia, R., Karki, S., Oliva, O., Hock, T., et al. (2013). Pleural mesothelial cell differentiation and invasion in fibrogenic lung injury. Am. J. Pathol. 182, 1239-1247. doi: 10.1016/j.ajpath.2012.12.030

Conflict of Interest Statement: The authors declare that the research was conducted in the absence of any commercial or financial relationships that could be construed as a potential conflict of interest.

Received: 27 May 2014; accepted: 10 July 2014; published online: 01 August 2014. Citation: Batra H and Antony VB (2014) The pleural mesothelium in development and disease. Front. Physiol. 5:284. doi: 10.3389/fphys.2014.00284

This article was submitted to Membrane Physiology and Membrane Biophysics, a section of the journal Frontiers in Physiology.

Copyright (c) 2014 Batra and Antony. This is an open-access article distributed under the terms of the Creative Commons Attribution License (CC BY). The use, distribution or reproduction in other forums is permitted, provided the original author(s) or licensor are credited and that the original publication in this journal is cited, in accordance with accepted academic practice. No use, distribution or reproduction is permitted which does not comply with these terms. 